\title{
Tumor bracketing and safety margin estimation using multimodal marker seeds: a proof of concept
}

\author{
Tessa Buckle \\ Patrick T. K. Chin \\ Nynke S. van den Berg \\ Claudette E. Loo \\ Wim Koops \\ Kenneth G. A. Gilhuijs \\ Fijs W. B. van Leeuwen \\ Departments of Radiology and Nuclear Medicine \\ Division of Diagnostic Oncology at the Netherlands \\ Cancer Institute-Antoni van Leeuwenhoek Hospital \\ 1066 CX Amsterdam, The Netherlands
}

\begin{abstract}
Accurate tumor excision is crucial in the locoregional treatment of cancer, and for this purpose, surgeons often rely on guide wires or radioactive markers for guidance toward the lesion. Further improvement may be obtained by adding optical guidance to currently used methods, in the form of intra-operative fluorescence imaging. To achieve such a multimodal approach, we have generated markers that can be used in a pre-, intra-, and post-operative setting, based on a cocktail of a dual-emissive inorganic dye, lipids, and pertechnetate. Phantom experiments demonstrate that these seeds can be placed accurately around a surrogate tumor using ultrasound. Three-dimensional bracketing provides delineation of the entire lesion. Combined with the multimodal nature, this provides the opportunity to predetermine the resection margins by validating the placement accuracy using multiple imaging modalities (namely, x ray, MRI, SPECT/CT, and ultrasound). The dual-emissive fluorescent properties of the dye provide the unique opportunity to intra-operatively estimate the depth of the seed in the tissue via multispectral imaging: emission green $\lambda_{\max }=520 \mathrm{~nm} \leqslant 5 \mathrm{~mm}$ penetration versus emission red $\lambda_{\max }=660 \mathrm{~nm} \leqslant 12 \mathrm{~mm}$ penetration. By using particles with different colors, the original geographic orientation of the excised tissue can be determined. (๑) 2010 Society of Photo-Optical Instrumentation Engineers. [DOI: 10.1117/1.3503955]
\end{abstract}

Keywords: multimodality imaging; fluorescence; surgical guidance; marker seeds; dyes.

Paper 10329RR received Jun. 16, 2010; revised manuscript received Aug. 31, 2010 accepted for publication Aug. 31, 2010; published online Oct. 27, 2010.

\section{Introduction}

One of the main reasons for locoregional treatment failure in oncology is the inability to fully excise the (primary) tumor during surgery. For example, complete resection of cancer is particularly difficult when the tumor is nonpalpable. In such instances, surgical guidance techniques that help optimize the excision are expected to have a positive influence on the clinical outcome.

Initial detection of nonpalpable lesions at pre-operative imaging [Fig. 1(a)] is often followed by image-guided markation of the lesion [Fig. 1(b)]. These procedures typically aim to mark the center of the lesion using guide-wire localization (WL), radio-guided occult lesion localization (ROLL), or radioactive seed localization (RSL). In WL, a hook is placed in the center of the tumor and attached to a guide-wire ${ }^{1,2}$ along which the surgeon can excise the tissue. In ROLL, a nonspecific radiotracer $\left({ }^{99 \mathrm{~m}} \mathrm{Tc}-\mathrm{NanoColl}\right.$ or sulfur-colloid $)$ is injected into the tumor prior to surgery. ${ }^{3}$ The radioactive signal is then used to acoustically localize the lesion intra-operatively using a gamma probe. Radioactive seed localization (RSL) provides an alternative radio guidance method to ROLL using titanium

Address all correspondence to: Fijs W. B. van Leeuwen, Departments of Radiology and Nuclear Medicine, Division of Diagnostic Oncology at the Netherlands Cancer Institute-Antoni van Leeuwenhoek Hospital, 1066 CX Amsterdam, The Netherlands.Tel: 31-205-126-084;E-mail: fw.v.leeuwen@nki.nl marker seeds filled with a small amount of radioactive iodine $\left({ }^{125} \mathrm{I}\right) .{ }^{4-7}$

Image-guided localization is typically carried out using $\mathrm{x}$-ray imaging [such as (stereotactic) mammography (XM)], ultrasonography (US), or magnetic resonance imaging (MRI). ${ }^{4,8}$ Although WL, ROLL, and RSL all aid in the localization of cancer during surgery, accurate marker placement remains crucial. Hence, accurate verification of the marker relative to the tumor location in one or multiple preoperative imaging modalities may be useful. This strategy requires the localization markers to be visible in the same modality that was used to visualize the tumor. Clearly, the latter may vary for different tumor types and sites.

Intra-operative fluorescence imaging is emerging as an optical detection method in surgical oncology. Fluorescent dyes such as fluorescein and indocyanine green (ICG) are used in several different applications in patients, mostly for superficial imaging of blood flow or lymph node detection. ${ }^{9-12}$ Surprisingly, the potential of this optical modality in tumorlocalization applications is currently unexplored. Nevertheless, it can be used to overcome an important shortcoming of the current acoustic radioguidance methodsnamely, by providing real-time optical guidance.

$1083-3668 / 2010 / 15(5) / 056021 / 8 / \$ 25.00$ ○ 2010 SPIE 


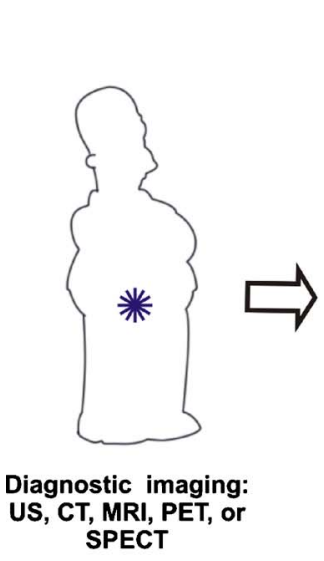

(a)
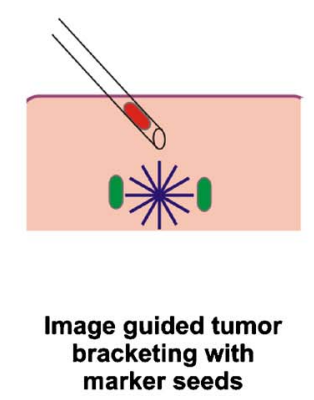

(b)

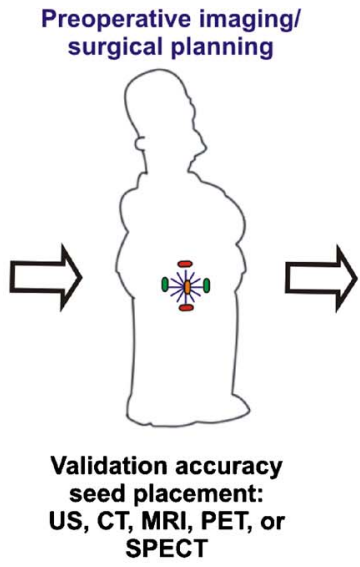

(c)

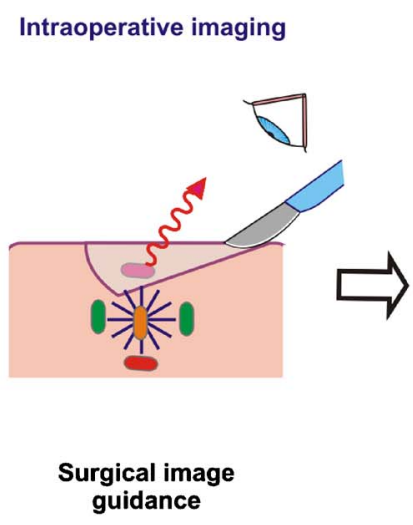

(d) Geographic
reorientation

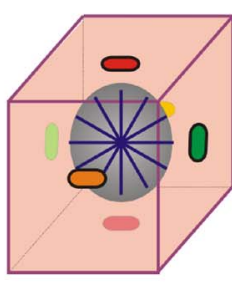

Pathology

(e)

Fig. 1 Schematic representation of the combined pre-, intra-, and post-operative imaging paradigm that can be achieved with multimodal marker seeds. (a) Tumor diagnosing imaging procedure. (b) Image-guided placement of bracketing tumor marker seeds. (c) Pre-operative validation of the marker placement accuracy. (d) Optical intra-operative detection of the tumor markers. (e) Geographical (re)orientation of the excised tissue section at pathology.

To eliminate the need for margin re-excision, it is vital that surgical resection margins do not contain any residual tumor tissue. For this reason, an additional small tumor-free margin around the tumor, the so-called surgical safety margin, is often excised. ${ }^{13,14}$ Different fluorescent wavelengths have different degrees of tissue penetration. While visual dyes (emission 400 to $600 \mathrm{~nm}$ ) can be detected only superficially, far-red and near-infrared dyes (emissions $\geqslant 650 \mathrm{~nm}$ ) give a much higher degree of tissue penetration, but can be detected only with a light-sensitive camera system. ${ }^{15}$ This difference in tissue penetration can be used to provide a rough estimate of the depth of the marker in the tissue. Multispectral fluorescence imaging thereby enables differentiation between simultaneous emissions at different wavelengths. ${ }^{16,17}$ Specifically for this purpose, we have previously developed dual-emissive (inorganic) dyes that have a visible green emission and a far-red emission that can be accurately detected without autofluorescence. ${ }^{18}$

Improved macroscopic localization methods may also be beneficial post-operatively at pathology. To report the presence of cancer on the resection edges back to the surgeon for potential re-excision, the pathologist typically indicates the direction in which the positive edge was observed. Commonly, India ink and sutures are used to mark the original orientation of the excised tissue in the patient. ${ }^{19}$ Threedimensional bracketing of a lesion with differently "colored" marker seeds may provide an opportunity to record the relative location and orientation of the tumor in individual patients.

The technical challenge we have set out to solve is the development of marker seeds that (1) can be accurately placed at different positions surrounding the lesion and thereby implementing a safety margin [Fig. 1(b)]; (2) can be preoperatively detected using the same imaging technique employed to diagnose the lesion rather than only with the modality used for bracketing [Fig. 1(c)]; (3) can be optically located during surgical procedures [Fig. 1(d)]; and (4) will provide information about specimen orientation for pathologists [Fig. 1(e)] after excision of the lesion. To incorporate all these properties into a single marker, we have generated multimodal marker seeds that are visible with fluorescence imaging, XM, US, MRI, computed tomography (CT), and singlephoton emission computed tomography (SPECT). We have studied their potential in providing surgical guidance in pre-, intra-, and post-operative phantom experiments and in experiments in dead surplus mice.

\section{Materials and methods}

\subsection{Dual-Emissive Dyes}

Lipid-coated InP/ZnS particles were synthesized and irradiated as described previously. ${ }^{18}$ These particles have two separate emissions-namely, an exciton emission at $\lambda_{e m}$ $=520 \mathrm{~nm}, 600 \mathrm{~nm}$, or $660 \mathrm{~nm}$ (short lifetime) and a defect emission at $\lambda_{\max }=660 \mathrm{~nm}$ [long lifetime $>60 \mathrm{~min}$; see Figs. 2(a) and 2(b)]. The latter emission can be preilluminated $\left(\lambda_{e m}=366 \mathrm{~nm}\right)$ and detected without real-time excitation.

For safety margin estimation, green particles $\left(\lambda_{e m}\right.$ $=520 \mathrm{~nm})$ were used. For geographic tissue orientation, green, orange $\left(\lambda_{e m}=600 \mathrm{~nm}\right)$, and red $\left(\lambda_{e m}=660 \mathrm{~nm}\right)$ particles were used. Herein, the colors were used for visual detection.

\subsection{Creation of Multimodal Marker Seeds}

Marker seeds were made of glass capillaries, which were melted and thereby closed at one end. The seed was then filled with a mixture of the dual-emissive $\mathrm{InP} / \mathrm{ZnS}$ QDs [see Fig. 2(c)], triton X-100 (10\%), and pertechnetate solution $\left({ }^{99 m} \mathrm{Tc}\right.$; $40 \mathrm{MBq} / 50 \mu \mathrm{l})$. The seed was closed with a small drop of epoxy resin, leaving a small air bubble inside. Total dimension of the seed was $1.0 \times 8.0 \mathrm{~mm}$ so that it fits in a hollow implantation needle.

\subsection{Generation Tumor Phantom and Seed Placement}

A ${ }^{125}$ I seed (STM1251, Bard Brachytherapy), situated in a tiny amount of silicone gel, was placed in the center of a piece of tenderloin to act as a tumor phantom. After preillumination, 


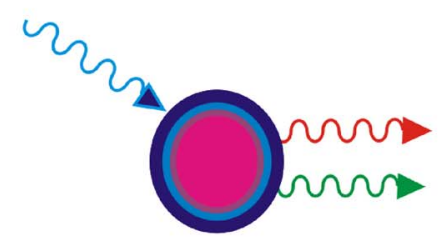

$$
\begin{aligned}
& =\lambda \mathrm{em} 660 \mathrm{~nm} \\
& =\lambda \mathrm{ex} \\
& =\lambda \mathrm{em} 520 \mathrm{~nm}
\end{aligned}
$$

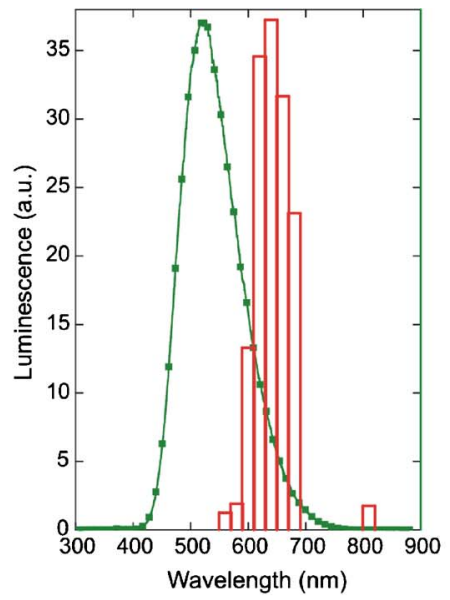

(b)

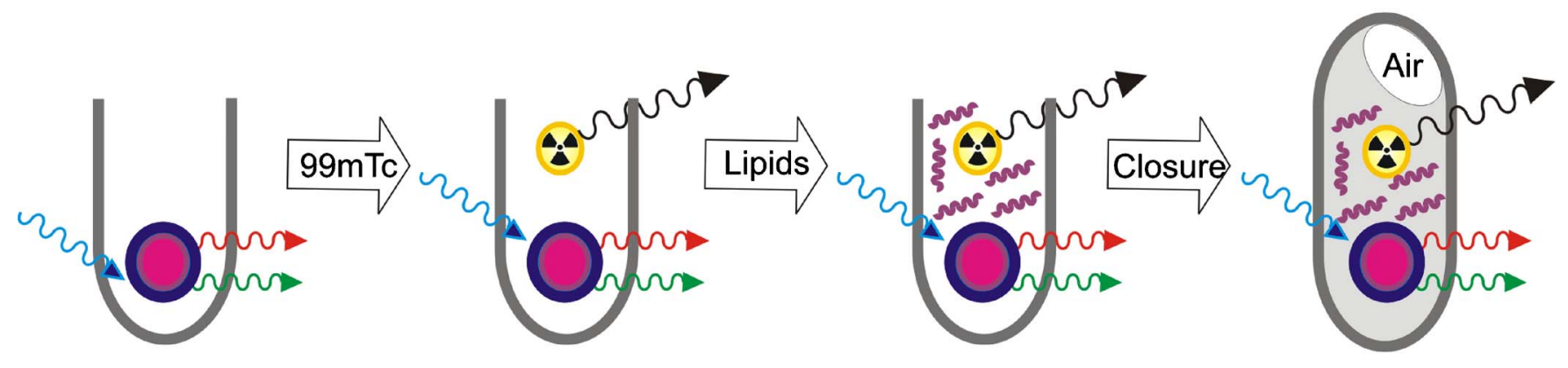

(c)

Fig. 2 Dual-emissive $\mathrm{InP} / \mathrm{ZnSn}$ particles and generation of multimodal marker seeds. (a) Schematic representation of dual-emissive InP/ZnS particles. (b) Normalized fluorescent emission spectra with $\lambda_{e m}=520 \mathrm{~nm}$ (exciton green) and $\lambda_{e m}=660 \mathrm{~nm}$ (defect; red). (c) Schematic generation of multimodal marker seeds that incorporate dual-emissive dyes and ${ }^{99 \mathrm{~m}} \mathrm{Tc}$ lipids. (Color online only.)

the multimodal marker seeds were placed at different positions surrounding the surrogate tumor under US guidance using a hollow implantation needle (AccuNeedle; 18 gauge, $20 \mathrm{~cm}$; Oncura). Alternatively, a seed was placed in the prostate of a surplus dead male mouse.

\subsection{Imaging Modalities}

\subsubsection{US}

Placement of the multimodal marker in the tissue phantom was conducted under US guidance by an experienced radiologist, using a Hitachi E7U-MT27-S1 US scanning device equipped with a linear probe (6 to $14 \mathrm{MHz}$ ) typically used for breast scanning.

\subsubsection{X-ray mammography}

The phantom tissue was positioned on a Perspex plate $(3 \mathrm{~mm}$ thick). The sample was imaged on an x-ray mammograph (Selenia, Lorad) at $25 \mathrm{kV}$ and $85 \mathrm{mAs}$, to provide anatomical detail and to visualize the multimodal seeds and surrogate tumor.

\subsubsection{MRI}

For MR imaging, the phantom was placed in a clinical Philips Achieva 3.0T MRI scanner (Philips). A standard scanning sequence was used to acquire T2-weighted images using a Flex
Small wrist coil. The imaging data was reconstructed using the Achieva scanner software. Kodak viewing software (Kodak) was used to analyze the images.

\subsubsection{SPECT/CT}

For visualization of the radioactivity inside the seeds, the phantom was placed in an animal holder (Equipment Veterinaire MINERVE) before performing a SPECT/CT scan on the nanoSPECT/CT (Bioscan, Inc.) A region of interest incorporating the whole phantom was selected, based on a (sagittal) tomographic planning $\mathrm{x}$-ray image, and a dual-isotope SPECT scan $\left({ }^{125} \mathrm{I}: 30 \mathrm{keV} ;{ }^{99 \mathrm{~m}} \mathrm{Tc}\right.$ : $\left.140 \mathrm{keV}\right)$ of the same field of view was initialized. Directly after the SPECT imaging sequence, a helical 3-D CT acquisition of the phantom was performed. After acquisition, the CT data was reconstructed with HiSPECT software (Scivis GmbH). The SPECT and CT data sets were automatically co-registered, and the images were analyzed using the InVivoScope post-processing software (Bioscan, Inc.)

\subsubsection{Optical spectroscopy and imaging}

UV-VIS spectra were recorded using a Perkin Elmer Lambda 900 or Lambda 3b spectrophotometer. Steady state fluorescence spectra were measured with an Edinburgh Instruments FS920 spectrophotometer $\left(\lambda_{e x}=450 \mathrm{~nm} ; \quad \lambda_{e m}=520 \mathrm{~nm}\right)$. 


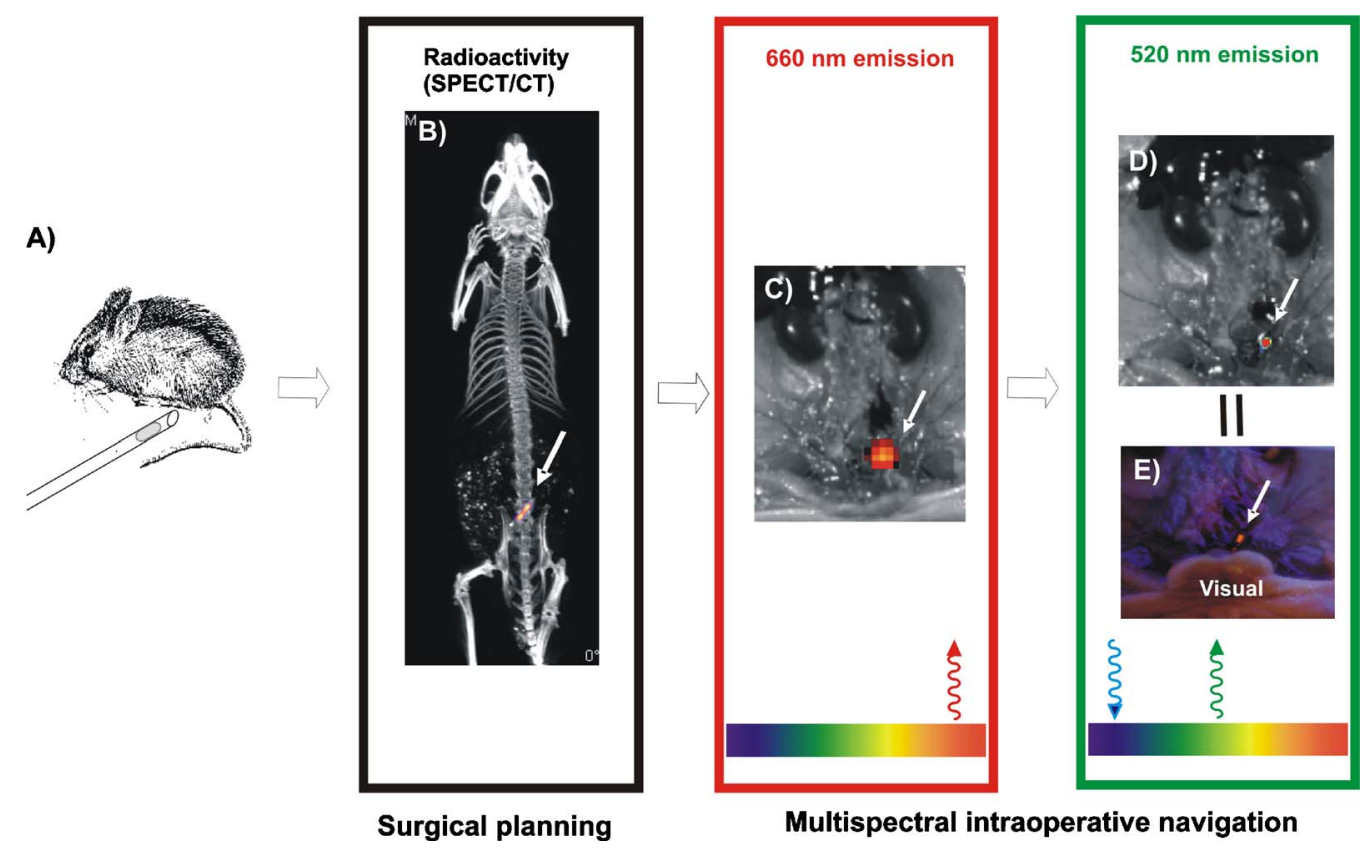

Fig. 3 Imaging of multimodal marker seeds: (a) Schematic representation of the placement of the seed in a mouse prostate. (b) 3-D SPECT/CT image visualizes the seed localization (color-coding: fire) with respect to the mouse anatomy, followed by (c) $660 \mathrm{~nm}$ emission (color-coding: glow), (d) $520 \mathrm{~nm}$ emission (color-coding: rainbow) and (e) a visual fluorescent (orange) image of the exposed marker seed. (Color online only.)

Long-term decay of the $\mathrm{InP} / \mathrm{ZnS}$ defect emission $\lambda_{\text {em }}$ $=660 \mathrm{~nm}$ was recorded using the IVIS 200 camera (Xenogen Corp.) using open filter settings $\left(\lambda_{e m}=515\right.$ to $\left.885 \mathrm{~nm}\right)$. The narrow bandpass filter set of the IVIS 200 system was used to record the defect spectrum of $\mathrm{InP} / \mathrm{ZnS}$ QDs in 1-octadecene $\left(\lambda_{e x}=366 \mathrm{~nm}, 4-\mathrm{W}\right.$ electrical power, UV light).

Tissues and mice were placed in the temperaturecontrolled $\left(37^{\circ} \mathrm{C}\right)$ imaging chamber of the IVIS 200. The exciton emission was measured with the standard GFP excitation and emission settings $\left(\lambda_{e x}=445\right.$ to $490 \mathrm{~nm} ; \lambda_{e m}$ $=515$ to $575 \mathrm{~nm}$; acquisition time: $30 \mathrm{~s}$ ). The defect emission was imaged with open filters (acquisition time: $120 \mathrm{~s}$ ). To determine the tissue penetration of the different emissions, the distance of the seed to the edge of the phantom was decreased by approximately $2 \mathrm{~mm}$ after every scan. The signal intensities (photons $/ \mathrm{cm}^{2} / \mathrm{s}$ ) were quantified with Living Image 3-D software (Xenogen Corp.). Signal-to-background ratios (SBRs) were calculated using the following formula: $S B R$ $=I_{Q D} / I_{\text {background }}$, where $I_{Q D}$ and $I_{\text {background }}$ are, respectively, the signal intensities from the exciton/defect emission of the $\mathrm{InP} / \mathrm{ZnS}$ QDs and the background signal from surrounding tissue at the respective imaging settings. An SBR ratio $>2$ was considered to be useful for diagnostic purposes. Tissue penetration experiments were conducted in triplicate.

\section{Results}

To combine the ability to pre-operatively set and validate preferred resection margins with optical detection and safety margin estimation, multimodal and dual-emissive marker seeds were generated. The potential of these marker seeds for pre-operative tumor bracketing, intra-operative surgical guidance, and post-operative geographic reorientation was studied.

To illustrate the multimodal seed concept and the potential of these seeds in vivo, seeds were first implanted into the prostate of a dead surplus mouse. After implantation with a hollow needle into the prostate, a SPECT/CT image was made, clearly visualizing the location of the radioactive seed with respect to the anatomy of the mouse (Fig. 3; SPECT/ $\mathrm{CT}$ ). Following the pre-operative SPECT/CT, intra-operative multispectral fluorescence imaging enabled detection of the seed (Fig. 3). Here, the defect emission allows detection without autofluorescence. ${ }^{18}$ In addition, the visual exciton emission of the InP/ZnS-particles in the seed could also be detected by eye after excitation with a UV light source [flashlight; Fig. 3(e)]. Due to the small size of the animal and relatively large size of the marker seed, these experiments unfortunately did not enable a form of surgical safety margin estimation.

The potential use of multimodal marker seeds in surgical safety margin estimation was further investigated in a tissue phantom using a surrogate tumor. After placement under US guidance [Figs. 4(a) and 4(bI)], the distinctive contrastgenerating properties of the seeds could be exploited to differentiate the seeds from their environment. T2-weighted MR imaging provided a clear distinction between the glass seed itself (black) and the lipid content of the seed [white; see Fig. 4(bII)]. With $x$ ray, the difference in density provides a clear distinction between the tissue, the silicone rubber, the titanium shell of the ${ }^{125} \mathrm{I}$-seed, and the glass of the multimodal marker seeds [Fig. 4(bIII)]. Dual-isotope SPECT/CT allowed differentiation between the ${ }^{125}$ I-seed and the ${ }^{99 \mathrm{~m}}$ Tc-containing multimodal marker seeds [see Fig. 4(bIV)].

To mimic intra-operative surgical safety margin estimation, the phantom was imaged in a multispectral manner at different stages during the resection. For a representation of the tissue penetration at the different emissions, see Fig. 5(a). 

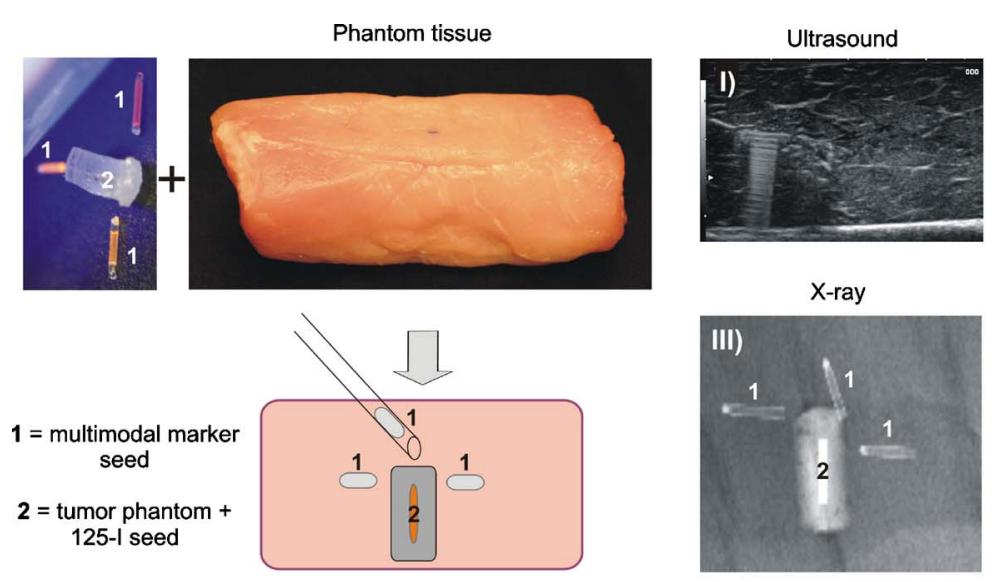

(a)
X-ray
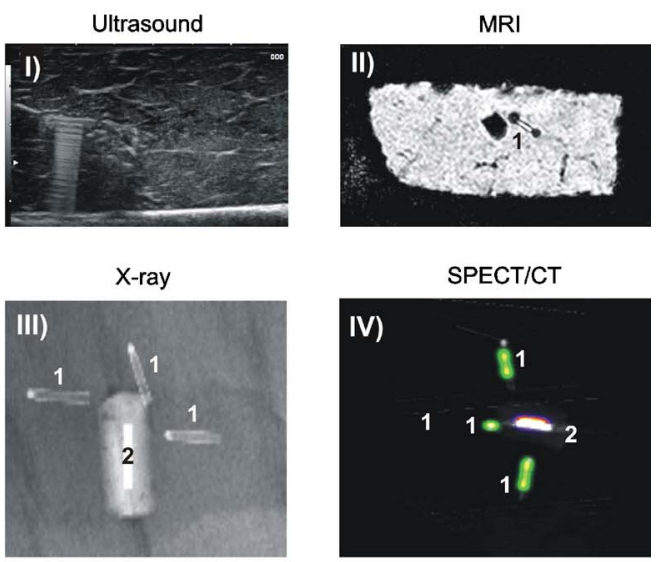

(b)

Fig. 4 "Pre-operative" multimodal seed bracketing and placement delineation in a tumor phantom. (a) 3-D tumor bracketing with multimodal marker seeds. Phantom contains a surrogate tumor in a fresh piece of tenderloin. (b) Pre-operative assessment of the marker seed localization using (I) ultrasound — the marker seeds deflect the ultrasound signal enabling accurate detection; (II) T2-weighted MRI—the marker seeds can be accurately discriminated from the surrounding by the lack of signal from the seed and the white signal from its contents; (III) a planar X-ray image-the high attenuation of the seed material is easily detected; and (IV) SPECT/CT—-dual-isotope SPECT enables differentiation between ${ }^{99 m}$ Tc-containing multimodal marker seeds (color-coding: green) and the ${ }^{125}$ I containing iodine seed (color-coding: fire). (Color online only.)

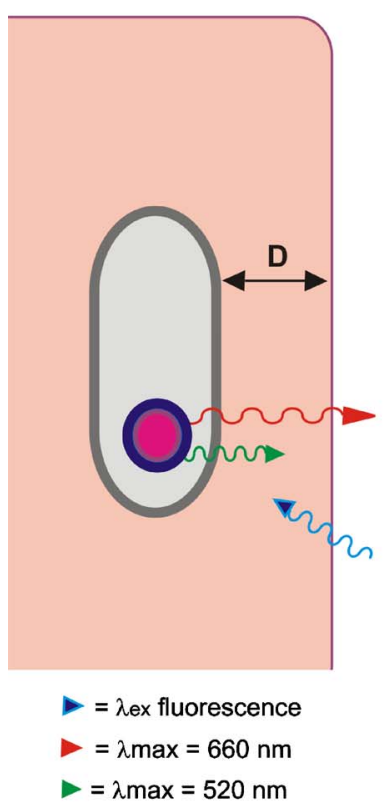

(a)

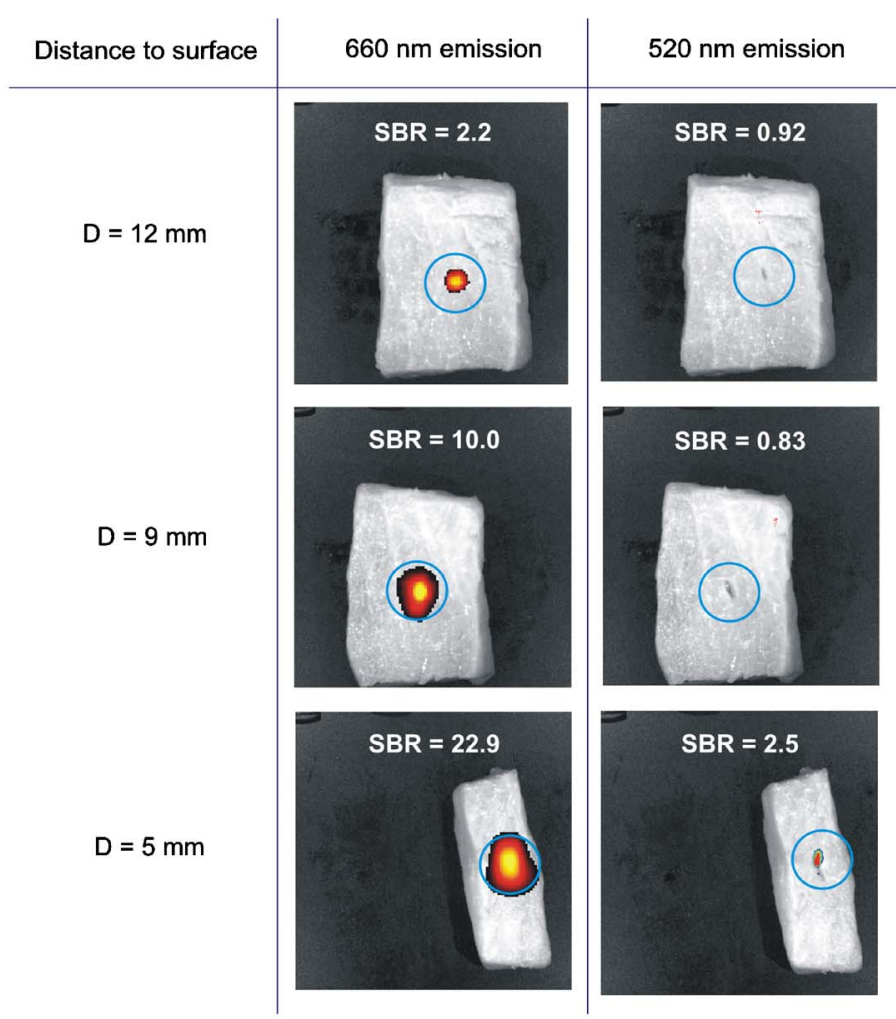

(b)

Fig. 5 Margin estimation based on multispectral imaging of dual-emissive seeds. (a) Schematic representation of the tissue penetration depth of the different optical emissions from the marker seeds; fluorescence excitation $\left(\lambda_{\text {ex }}=366 \mathrm{~nm}\right.$; blue) and the emissions $\lambda_{\max }=520 \mathrm{~nm}$ (green) and $\lambda_{\max }$ $=660 \mathrm{~nm}$ (red). (b) Detection of $660 \mathrm{~nm}$ (color-coding: glow) and $520 \mathrm{~nm}$ (color-coding; rainbow) emissions during the layer-by-layer exposure of the marker seeds. Fluorescent signal intensities are depicted as signal-to-background ratio (SBR). (Color online only.) 


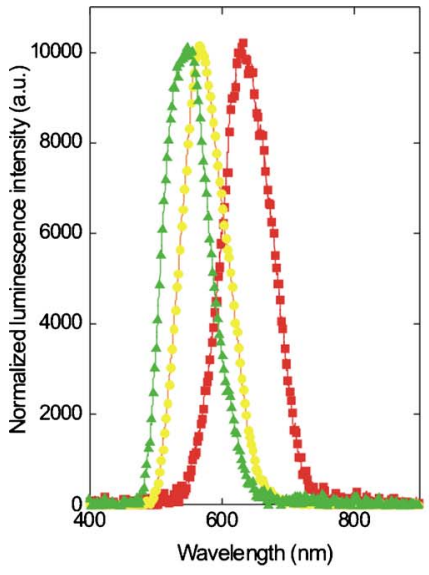

(a)

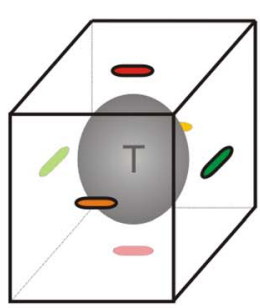

(b)

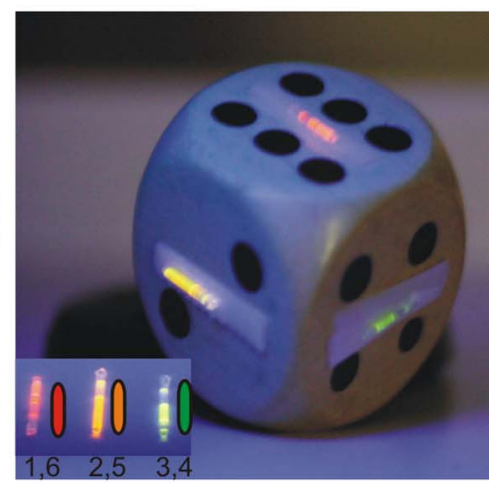

(c)

Fig. 6 3-D tumor bracketing for (re)orientation at pathology: (a) Differences in emission peaks of the different InP/ZnS particles. (b) Schematic representation of 3-D tumor bracketing with differently colored seeds (green/orange/red). (c) Proof of concept on a die allowing accurate visual identification and differentiation of the marker seeds. (Color online only.)

Signal-to-background ratios (SBRs) were used to present the ability to detect the different emissions at a different distance from the seed to the surface. The preilluminated defect emission could already be detected while the seed was situated at a distance of $12 \mathrm{~mm}(\mathrm{SBR}=2.2)$, followed by a ten-fold increased SBR at a distance of $5 \mathrm{~mm}$ [Fig. 5(b)]. In contrast, the exciton signal could be detected only starting at a distance to the surface of $5 \mathrm{~mm}(\mathrm{SBR}=2.5)$.

To exploit the value of the fluorescence detection in geographical pathologic guidance, seeds filled with differently colored $\mathrm{InP} / \mathrm{ZnS}$ particles were used. These particles emit light at different wavelengths and thus have different visual colors: green: $520 \mathrm{~nm}$; orange: $600 \mathrm{~nm}$; and red: $660 \mathrm{~nm}$ [see Fig. 6(a)]. To prove the concept of using 3-D bracketing with marker seeds in geographic tissue (re)orientation, the differently colored seeds were placed on the different sides of a dice. Seeds filled with red particles were placed at position one and six, seeds filled with orange particles were placed at the two and five position, and seeds filled with green particles were placed at position three and four of the dice [Figs. 6(b) and $6(\mathrm{c})]$. When placed under a UV light source, it was possible to visually discriminate between the different orientations. The same differentiation is of course possible using different settings on a (multispectral) fluorescence camera.

\section{Discussion}

In the clinic, tumor-lesions are often marked before surgical removal. Multimodal markers may extend the use of current tumor marking approaches, as they are applicable during the entire pre-, intra-, and post-operative trajectory. The different properties of our experimental multimodal seeds can be used for (1) accurate seed placement, (2) verification of the placement accuracy, (3) fluorescence guided excision with surgical safety-margins, and (4) ex vivo verification of original specimen orientation.

\subsection{Clinical Application Seed-Based Tumor Bracketing}

Pleijhuis et al. ${ }^{1}$ have stated that the restricted visibility of the lesion during excision could explain the high rate of positive resection margins reported in the literature. Although not yet widely applied, one approach to better delineate the outline of larger and more complex lesions is pre-operative placement of markers bracketing the tumor in three dimensions (3-D). ${ }^{2,5}$ Without increasing the need for re-excision, tumor bracketing can reduce the amount of healthy breast tissue that is unnecessarily removed during tumor excision. ${ }^{20}$ Bracketing with radioactive seeds has been reported to be of value for nonpalpable breast tumors such as invasive ductal/lobular carcinoma, ductal carcinoma in situ, and papillary carcinoma. ${ }^{4,5,7}$ Evidently, the here-reported multimodal seeds can potentially be used in previously reported applications for RSL. An advantage of marker seeds and guidewires over ROLL is that the first two do not suffer from tracer spillage and migration. ${ }^{1}$ We envision that marker seeds also have more potential in abdominal applications where WL-bracketing is cumbersome; WL-bracketing restricts the surgical access and can be manipulated during patient handling. ${ }^{1,4}$ Furthermore, the use of, e.g., brachytherapy ${ }^{125} \mathrm{I}$-seeds in the prostate is already widely applied. ${ }^{21}$

\subsection{Image-Guided Bracketing and Verification of the Placement Accuracy}

In the phantom experiments described, seeds were placed at a predetermined position under US guidance. US is one of the modalities frequently used for placement of (bracketing) markers under real-time guidance [Fig. 1(b)]., ${ }^{4,6}$ Although not studied by us, placement under guidance of $\mathrm{x}$ ray $(\mathrm{CT})$, SPECT/CT, and MRI is also a possibility with these seeds, as their multimodal character also provides contrast in these modalities (see Fig. 4). Detection of the marker seeds using the same modality that was used to detect the tumor itself [XM, MRI, or SPECT/CT; Fig. 1(a)] can also be achieved due to the 
multimodal nature of the marker seeds described here (see Fig. 4). This allows for accurate validation of their placement with respect to the tumor edge and surgical margins. For example, when one of the markers is misplaced, the surgical procedure can be slightly redirected during the planning phase [see Fig. 1(c)].

\subsection{Surgical Guidance}

The dual-emissive optical properties of the seeds provide the unique opportunity to optically determine the position of the marker seed during the intervention via real-time multispectral fluorescence imaging [Fig. 1(d)]. The differences in tissue penetration between the separate emissions also offer information about the obtained surgical safety margin (see Fig. 5). Here, sole detection of the $660 \mathrm{~nm}$ emission suggests that the tumor is located $\leqslant 12 \mathrm{~mm}$ below the surface, whereas detection of both emissions (520 and $660 \mathrm{~nm})$ suggests that the seed is located $\leqslant 5 \mathrm{~mm}$ below the surface. Hence, when only the $660 \mathrm{~nm}$ emission is detected, a surgical safety margin of $>5 \mathrm{~mm}$ is retained. This "traffic light approach" underlines the potential of dual-emissive optical properties in a single marker seed.

\subsection{Post-Operative Geographic Guidance}

After surgical planning and excision, histological analysis is performed to assess possible presence of tumor in the resection margins. Herein, geographic orientation of the excised specimen relative to the pre-operative image and the patient is critical. Currently, excised tissue is marked with India ink or sutures. ${ }^{19}$ When microscopic evaluation of the tissue shows positive resection margins, the location of these markings provides a rough estimate of the location at which more tissue should be excised. Three-dimensional bracketing of a lesion with differently colored marker seeds can be used as an alternative macroscopic marking method. The three-dimensional nature could provide more accurate geographical navigation. We have shown that a color scheme can be used to differentiate between different geographic locations [Fig. 1(e) and Fig. 6]. It must be noted that accidental damage of the marker seeds could possibly contaminate sectioning tools with dyes and radioactivity. Moreover, their use cannot replace microscopic analysis; it can merely help guide a pathologist to the regions that require detailed analysis.

\subsection{Marker Seeds as a Versatile Multimodal Container}

We have demonstrated that marker seeds can be used as containers that incorporate multiple contrast generating media and have utilized a number of such media viz dual-emissive dyes, ${ }^{99 \mathrm{~m}} \mathrm{Tc}$, and lipids. However, the variations that can be achieved with such containers are endless and mostly depend on the desire of the user. For example, the incorporation of isotopes with a different energy window provides the possibility to detect seeds with different modalities, e.g., PET, or to distinguish individual seeds using dual-isotope SPECT [see Fig. 4(bI)]. The latter can be combined with the use of differently colored dyes, improving the assessment of specimen orientation even further. Other contrast agents can also be incorporated to help improve the visualization/differentiation in the different modalities, e.g., paramagnetic contrast for MRI or alternative dyes.

\subsection{Clinical Translation}

With the described phantom data, we demonstrated the potential use of multimodal marker seeds in tumor bracketing applications. We realize however, that the described seeds are not swiftly translatable to a clinical application. In our opinion, the main limiting effects herein are (1) seed material, (2) the seed contents (the fluorescent component), and (3) realtime intra-operative (multispectral) fluorescence detection.

For a more straightforward clinical translation, perhaps the material of the outer shell of the seed should be adapted. Where the epoxy resin sealed glass seeds provided a relatively easy to produce capsule for our proof-of-concept studies, other materials, e.g., polymer based are also a possibility. Desirable properties for such encapsulating seed materials are transparency and rigidity. The latter will prevent spillage.

A major benefit of the marker seed approach is that the seeds will be removed during the surgical intervention. In combination with the confinement of a closed marker seed, this can aid in the translation of new contrast-generating media that have not yet been approved for (intravenous) clinical exposure. This said, rapid approval for a clinical use is probably most easily obtained using already clinically applied contrast media. In our case, the dual-emissive inorganic dyes used are not clinically approved. We have previously shown, however, that the clinically applied dyes fluorescein and ICG give tissue penetration similar to the two emissions of our dualemissive inorganic dye. ${ }^{18}$ Hence, a mixture of fluorescein and ICG can perhaps also be used in such an application.

Ideally, intra-operative visualization should be performed with fast data acquisition. This requires a good SBR and a high sensitivity for the surgical fluorescence cameras. At present, the fairly long acquisition time needed to detect the defect-emission with the IVIS system (120 s) compared to the time needed to detect the exciton emission (30 s) slows down the acquisition process. Dyes with stronger emission intensities are expected to improve the detection sensitivity. Acquisition, however, also depends highly on the sensitivity of the camera system. Preferably, dye-camera combinations are specifically tailored.

\section{Conclusions}

We have provided a proof of concept for using multimodal marker seeds in tumor bracketing and surgical guidance. Using the unique multimodal properties of the seeds, placement accuracy can be validated and resection margins can be predetermined. Moreover, multispectral imaging of the dualemissive fluorescent content provides the opportunity for estimation of surgical safety margins. Last, the differently colored fluorescent particles provide geographical information to the pathologist regarding the original orientation of the lesion in the patient.

\section{Acknowledgments}

This research is supported, in part, by a KWF-translational research award (Grant No. PGF 2009-4344; FvL) and via FP7-HYPERImage (Grant No. 201651). 


\section{References}

1. R. G. Pleijhuis, M. Graafland, J. de Vries, J. Bart, J. S. de Jong, and G. M. van Dam, "Obtaining adequate surgical margins in breastconserving therapy for patients with early stage breast cancer: current modalities and future directions," Ann. Surg. Oncol. 16, 2717-2730 (2009).

2. L. Liberman, J. Kaplan, K. J. Van Zee, E. A. Morris, L. R. LaTrenta, A. F. Abramson, and D. D. Dershaw, "Bracketing wires for preoperative breast needle localization," Am. J. Roentgenol. 177, 565572 (2001).

3. V. Lavoué, C. Nos, K. B. Clough, F. Baghaie, E. Zerbib, B. Poulet, M.-A. Lefrère Belda, A. Ducellier, and F. Lecuru, "Simplified technique of radioguided occult lesion (ROLL) plus sentinel lymph node biopsy (SNOLL) in breast carcinoma," Ann. Surg. Oncol. 15(9), 2556-2561 (2008).

4. R. J. Gray, B. A. Pockaj, P. J. Karsteadt, and M. C. Roarke, "Radioactive seed localization of nonpalpable breast lesions is better than wire localization," Am. J. Surg. 188, 377-380 (2004).

5. C. E. Cox, B. Furman, N. Stowell, M. Ebert, J. Clark, E. Dupont, A. Shons, C. Berman, J. Beauchamps, M. Gardner, M. Hersch, P. Venugopal, M. Szabunio, J. Cressman, N. Diaz, V. Vrcel, and R. Fairclough, "Radioactive seed localization breast biopsy and lumpectomy: can specimen radiographs be eliminated?" Ann. Surg. Oncol. 10(9), 1029-1047 (2003).

6. M. D. Fleming, B. A. Pockaj, A. J. Hansen, R. J. Gray, and M. D Patel, "Radioactive seed localization for excision of nonpalpable intransit metastatic melanoma," Radiol. Case Rep. 1(2), 54-57 (2006).

7. J. H. Hughes, M. C. Mason, R. J. Gray, S. A. McLaughlin, A. C. Degnim, J. T. Fulmer, B. A. Pockaj, P. J. Karsteadt, and M. C. Roarke, "A multi-site validation trial of radioactive seed localization as an alternative to wire localization," Breast J. 14(2), 153-157 (2008).

8. T. Alderliesten, C. Loo, A. Paape, S. Muller, E. Rutgers, M. J. Vrancken-Peeters, and K. G. A. Gilhuijs, "On the feasibility of MRIguided navigation to demarcate breast cancer for breast-conserving surgery," Med. Phys. 37(6), 2617-2626 (2010).

9. Z. Alsagoff, P. T. K. Chew, C. K. L. Chee, J.-S. Wong, and T. Aung, "Indocyanine green anterior segment angiography for studying conjunctival vascular changes after trabeculectomy," Clin. Exp. Ophthal- mol. 29, 22-26 (2001).

10. N. Tagaya, R. Yamazaki, A. Nakagawa, A. Abe, K. Hamada, and T. Oyama, "Intra-operative identification of sentinel lymph nodes by near-infrared fluorescence imaging in patients with breast cancer," Am. J. Surg. 195(6), 850-853 (2008).

11. T. Kitai, T. Inomoto, M. Miwa, and T. Shikayama, "Fluorescence navigation with indocyanine green for detecting sentinel lymph nodes in breast cancer," Breast Cancer 12(3), 211-215 (2005).

12. H. Terasaki, Y. Miyake, and S. Awaya, "Fluorescein angiography of peripheral retina and pars plana during vitrectomy for proliferative diabetic retinopathy," Am. J. Ophthalmol. 123(3), 370-376 (1997).

13. K. von Smitten, "Margin status after breast-conserving treatment of breast cancer: how much free margin is enough?" J. Surg. Oncol. 98 , 585-587 (2008).

14. A. Luini, J. Rososchansky, G. Gatti, S. Zurrida, P. Caldarella, G. Viale, G. R. dos Santos, and A. Frasson, "The surgical margin status after breast-conserving surgery: discussion of an open issue," Breast Cancer Res. Treat. 113, 397-402 (2009).

15. A. Soubret and V. Ntziachristos, "Fluorescence tomography in the presence of background fluorescence," Phys. Med. Biol. 51, $3983-$ 4001 (2006).

16. L. Zhou and W. S. El-Deiry, "Multispectral fluorescence imaging," J. Nucl. Med. 50, 1563-1566 (2009).

17. R. M. Levenson and J. R. Mansfield, "Multispectral imaging in biology and medicine: slices of life," Cytometry, Part A 69A, 748-758 (2006).

18. P. T. K. Chin, T. Buckle, A. Aguirre de Miguel, S. C. J. Meskers, R. Jansen, and F. W. B. van Leeuwen, "Dual-emissive quantum dots for multispectral intra-operative fluorescence imaging," Biomaterials 31(26), 6823-6832 (2010).

19. S. E. Singletary, "Surgical margins in patients with early-staged breast cancer treated with breast conservation therapy," Am. J. Surg. 184, 383-393 (2002).

20. H. S. Burkholder, L. E. Witherspoon, R. P. Burns, J. S. Horn, and M. D. Biderman, "Breast surgery techniques: pre-operative bracketing wire localization by surgeons," Am. Surg. 73, 574-579 (2007).

21. M. M. Poggi, D. A. Grant, W. Sewchand, and W. B. Warlick, "Marker seed migration in prostate localization," Int. J. Radiat. Oncol., Biol., Phys. 56(5), 1248-2151 (2003). 